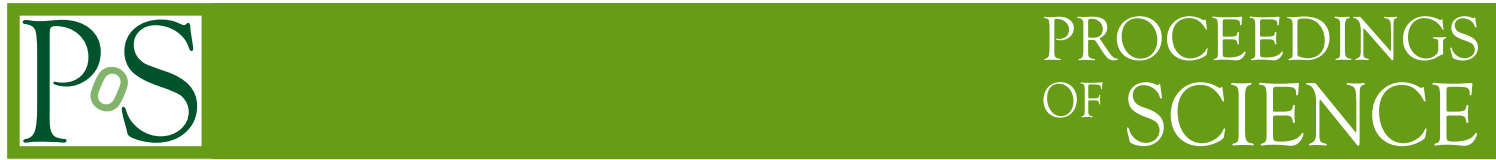

\title{
Spectral and QPO Properties of GRO J1655-40 in the 2005 Outburst
}

\section{Sandip K. Chakrabarti*}

S.N. Bose National Centre for Basic Sciences and Centre for Space Physics

E-mail: chakraba@bose.res.in

\author{
A. Nandi, D. Debnath, R. Sarkar, B. G. Dutta \\ Centre for Space Physics \\ E-mail: anujecsp.res.in, dipak@csp.res.in, \\ ritabrata@csp.res.in, broja@csp.res.in
}

GRO J1655-40 showed renewed X-ray activity in late February, 2005. We study the spectral properties and the Quasi-Periodic Oscillations (QPO) properties of this object for the first two weeks of RXTE observations after the outburst started. We show that as the spectrum becomes softer with time, the QPO frequency goes up. We analysed the QPOs for the period 25th Feb. 2005 to 12th of March, 2005 and showed that the frequency of QPO increased monotonically from $0.082 \mathrm{~Hz}$ to $15.01 \mathrm{~Hz}$. This agrees well if the oscillating shock is assumed to propagate with a constant velocity of about $1.9 \mathrm{~km} \mathrm{~s}^{-1} \mathrm{~s}$.

VI Microquasar Workshop: Microquasars and Beyond

September 18-22 2006

Società del Casino, Como, Italy

\footnotetext{
${ }^{*}$ Speaker.
} 


\section{Introduction}

GRO J1655-40 had been dormant since 1997 before suddenly coming to life in March, 2005 (Homan, 2005). We examine its behaviour in the first two weeks of RXTE observations after the begining of the outburst. We find that we can explain the features in the outburst in the same way as we did for other objects (Chakrababrti, 1998) using two component advective flow model (TCAF). After a long quiescence state in which the disk was practically absent, a fresh wave of matter is expected to produce a propagating shock wave as the matter moves towards the black hole. The spectral and timing behaviour of this object seem to conform with such a scenario. As the shock moves towards the black hole, the spectrum becomes softer as the post-shock region (CENtrifugal pressure supported BOundary Layer or CENBOL) cools down due to interception of increasing number of soft photons (Chakrbarti, 1997). At the same time, the infall time from the post-shock region is decreased and the QPO frequency, which is the inverse of the infall time, rises.

The oscillation of the shock model seems to explain all major aspects of the quasi-periodic oscillations and the QPO frequency is found to be generally related to the infall time $t_{i f}=R x_{s}\left(x_{s}-1\right)^{1 / 2}$, where $R$ is the shock compression ratio $\sim 4$ and $x_{s}$ is the instantaneous location of the shock. In case the shock propagates towards the black hole, the frequency is expected to rise monotonically. It would be of interest if we can show (a) that the QPOs are indeed from shock oscillations and (b) shock frequency increases monotonically according to the shock propagation model. In Chakrabarti \& Manickam (2000) and Rao et al. (2000), it was shown that another black hole candidate GRS 1915+105 had exhibited QPOs where the shock oscillation model was found to be valid. During the 1996/1997 outburst of GRO J1655-40, Remillard et al. (1999) also reported energy dependence of high frequency QPOs although no satisfactory model was discussed which could explain these behaviours.

\section{Observations, Results and Discussions}

In Chakrabarti \& Mandal (this volume and references therein) a brief introduction to how spectra and QPOs are generated has been presented. In the present paper, we analysed the data of the recent outburst using the PUBLIC data of the RXTE satellite. In order to quantify the nature of the spectra, it is customary to plot the hardness ratios. We extracted the photon counts in three bins with Channel numbers 0-7 (2-3.5keV), 8-24 (3.5-10.5keV) and 25-138 (10.5-60keV) respectively (denoted as $\mathrm{A}, \mathrm{B}$ and $\mathrm{C}$ respectively) and saved them in $1 \mathrm{~s}$ time bins. The ratios $H R 1=B / A$ and $H R 2=B / C$ will then give an idea about how the spectrum is changing with time. In Fig. 1 we show the variation of $H R 2$ as a function of $H R 1$ from 25th Feb. 2005 to 12th Mar. 2005. We note that the HR1 is monotonically decreasing while HR2 is monotonically rising during this period. Both of these behaviours give an indication of the increasing influence of the Keplerian disk in the flow. In the same figure, we also wrote down the observational IDs and the QPO frequencies.

In Fig. 2 we show the spectral evolution during this period. Initially, the spectral slope remains almost constant, although the intensity rises. In the last three days the spectral slope and the soft $\mathrm{X}$-ray intensity rapidly rises.

In Fig. 3, we plot the daily variation of power density spectra. As the spectrum softens the QPO frequency also rises. Both of these features are indications of the fact that the oscillating 


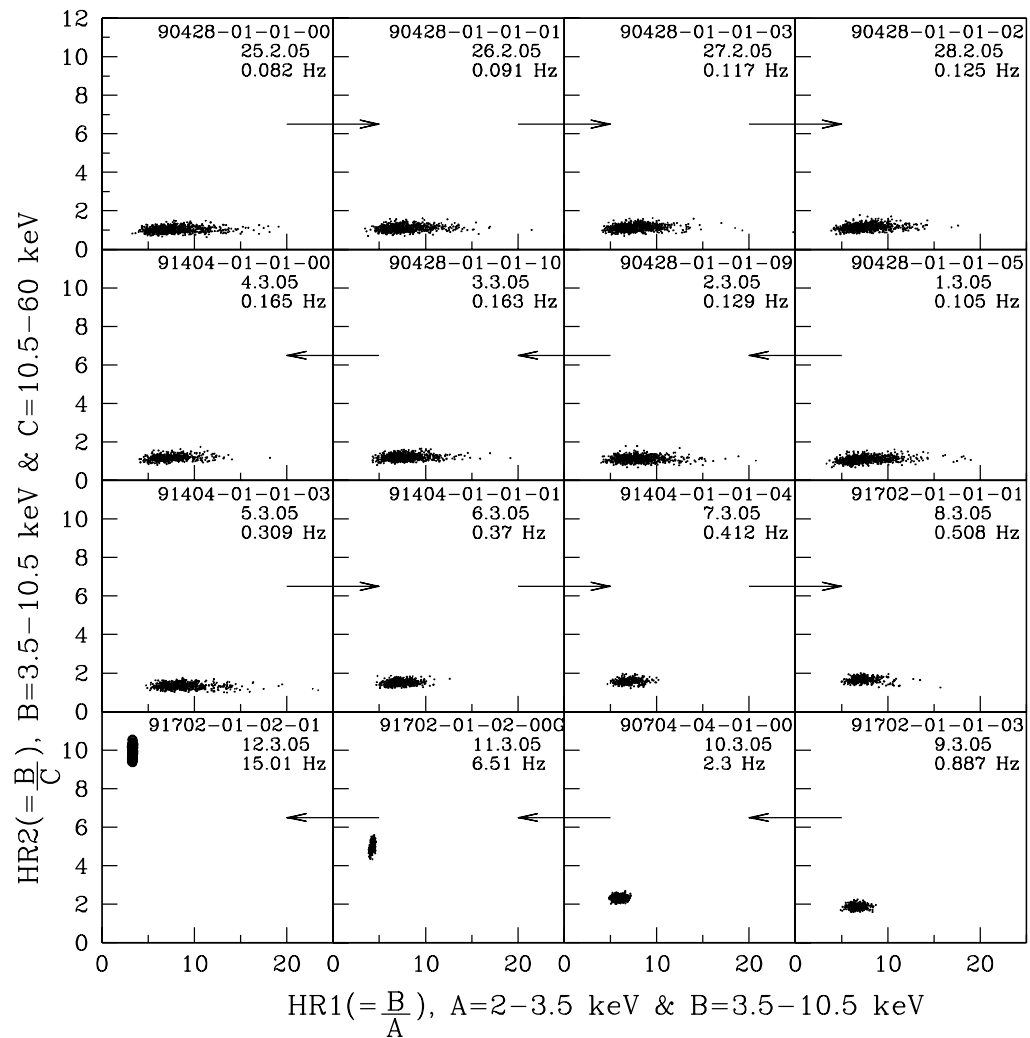

Figure 1: Variation of the hardness ratios during first two weeks of the outburst. We clearly see the dominance of the soft-photons as time passes by. The Observation IDs and the QPO frequencies have also been written down.

region which is causing QPO and is also responsible for producing the Comptonized radiation is moving towards the black hole. A few $\mathrm{mHz}$ produced on the first day becomes tens of $\mathrm{Hz}$ in two weeks time.

If we identify the frequency to be due to the oscillations from a strong shock, then it is easy to plot the theoretical variation of the QPO frequency with time. In Fig. 4, we plot this variation (solid curve) and also the observed QPO frequencies in solid circles. We assumed the shock to be moving at a constant velocity $v_{0}$ (the flow may itself move at a normal velocity). On the 12th of March, 2005 the peak is not very prominent but it can be shown to be present. The time measured from the observation of 25/2/05 when the shock was at $x_{s}=x_{s 0}$ is given by $t=\left(x_{s 0}-x_{s}\right) / v_{0}$. (Here all the radial distances are measured in units of the Schwarzschild radii). The frequency of QPO is already mentioned to be $v_{s}=\frac{\beta}{x_{s}\left(x_{s}-1\right)^{1 / 2}}$, where, $\beta \sim 1 / R \sim 0.25$ is the inverse of the shock strength $R$. The observed frequency $v_{s 0}=0.082 \mathrm{~Hz}$ at $t=0$ directly gives $x_{s 0}=1200$ as the launching radius. The constraint on the disappearance of the QPO on the latter half of the 12th of March, 2005, $(\sim 15.6 \mathrm{~d}$ from the beginning of launching), gives a very slow rate of shock propagation and the velocity is $v_{0} \sim 1.9 \mathrm{~km} \mathrm{~s}^{-1}$. However some variation in the shock strength along with a variation of the propagation velocity cannot be ruled out. 


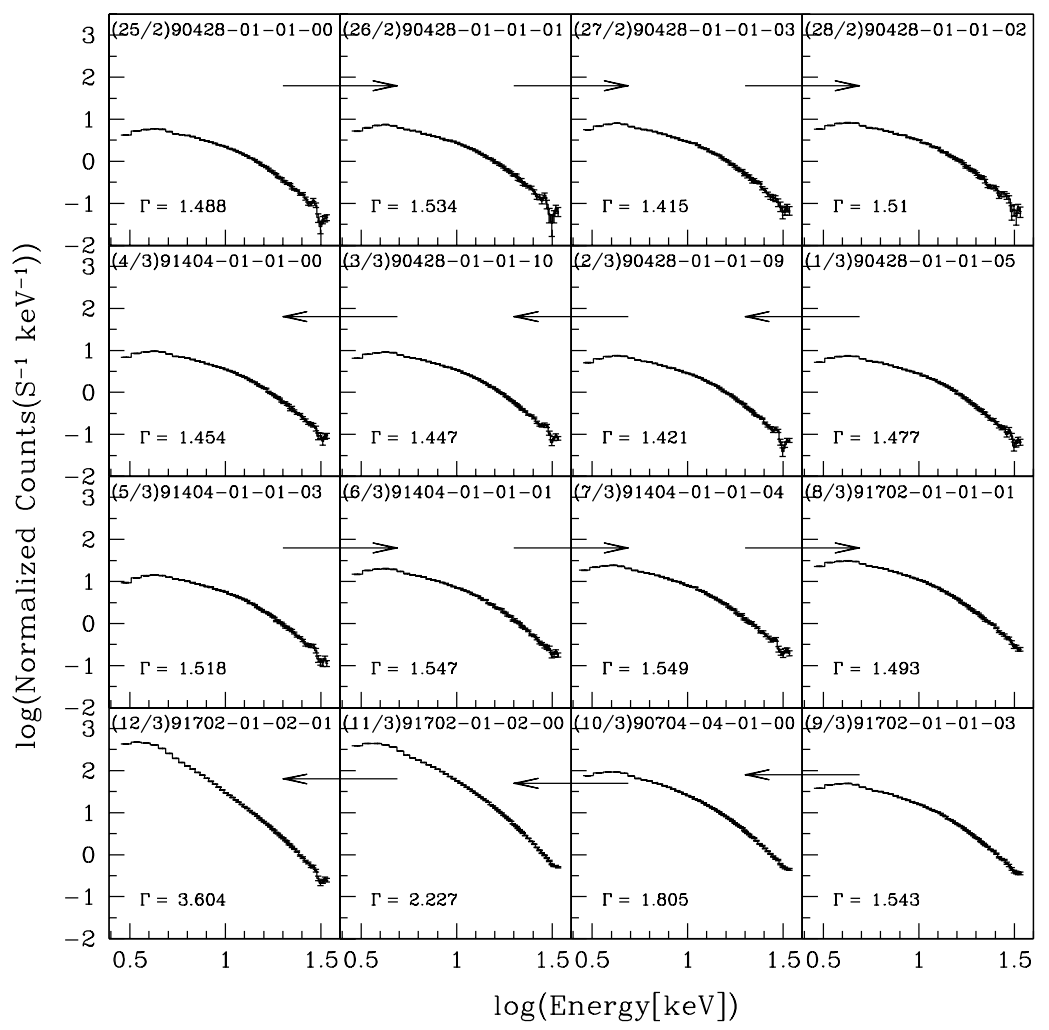

Figure 2: Variation of the spectra during 25th Feb. 2005 to 12th Mar. 2005. Though the spectral slope remains more or less constant for the first twelve days or so, the intensity of radiation increasing steadily. Subsequently, the spectral slope and the intensity both increase montonically.

\section{Concluding Remarks}

In this paper, we studied the evolution of the spectral properties (intensity, spectral index) and the QPO frequency of GRO J1655-40 during the first two weeks of the outburst. We showed that the spectrum becomes softer and the QPO frequency becomes higher. This is in line with the shock propagation model at the onset of the outburst. At each radius the shock also oscillates at a time scale comparable with the infall time scale from that radius. This consideration is able to explain the variation very easily.

\section{References}

[1] Chakrabarti, S.K., 1997, ApJ, 484, 313.

[2] Chakrabarti, S.K., 1998 in 'Observational Evidence for Black Holes in the Universe', ed. S.K. Chakrabarti, Kluwer Academic Press (Dordrecht).

[3] Chakrabarti, S.K., Acharyya, K. and Molteni, D., Astron. Astrophys., 4211 (2004)

[4] Chakrabarti, S.K. and Manickam, S.G., Astrophys. J. 531, L41 (2000)

[5] Chakrabarti, S.K. and Nandi, A. Indian J. Phys. 75(B), 1 (2000)

[6] Homan, J., Astron. Tel. ATEL No. 440 (2005) 


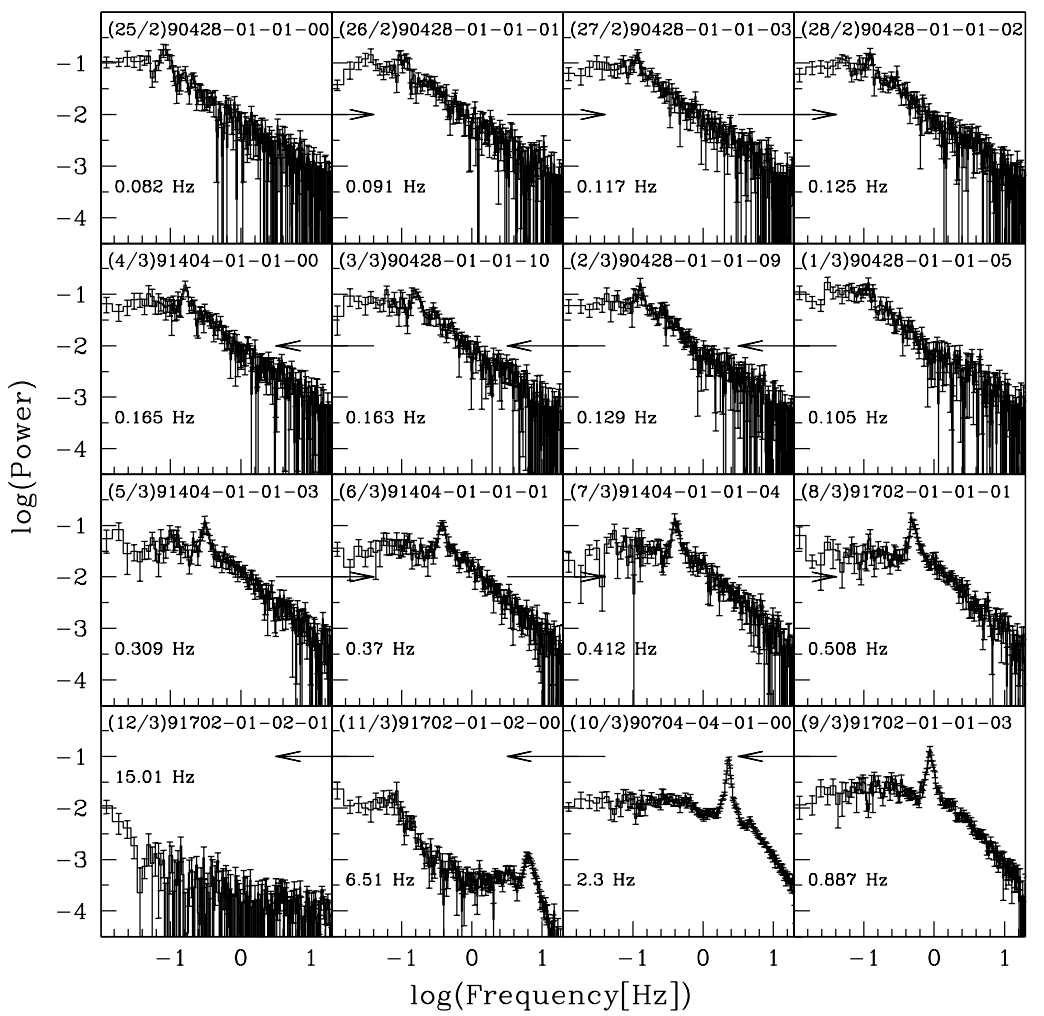

Figure 3: Variation of QPO frequency during the first two weeks of the outburst. The spectral softening, together with the increase of QPO frequency fits well with the oscillating shock model which is also propagating towards the black hole.

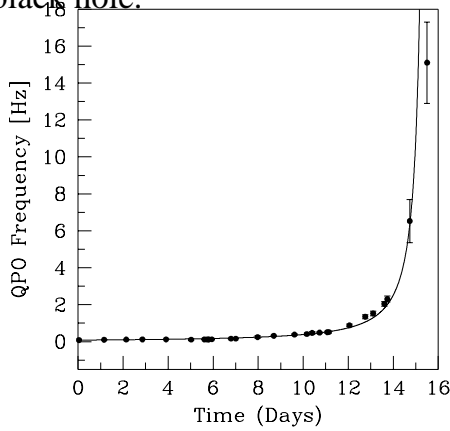

Figure 4: The observed QPO frequencies (circles) fit very well with an oscillating shock which is moving at a constant velocity towards the black hole (solid curve).

[7] Molteni, D., Sponholz, H. and Chakrabarti, S.K., Astrophys. J., 457, 805 (1996)

[8] Nandi, A., Manickam, S.G. and Chakrabarti, S.K., Indian J. Phys., 74(B), 331 (2000)

[9] Rao, A.R., Naik, S., Vadawale, S.V. and Chakrabarti, S.K., Astron. Astrophys., 360, L25 (2000)

[10] Remillard, R.E., Morgan, E.H., McClintock, J.E., Bailyn, C.D. and Orosz, J. A., Astrophys. J., 522, 397 (1999) 